\title{
Buenas prácticas ganaderas en Tauramena, Colombia
}

\section{Good farming practices in Tauramena, Colombia}

\author{
Sanabria Parrado Yury Shirley \\ ${ }^{1}$ Médico Veterinario Zootecnista, Universidad de los Llanos \\ yury.sanabria@unillanos.edu.co
}

Recibido 10 de Mayo 2016, Aceptado 29 de Octubre 2016

\section{RESUMEN}

Las exigencias de la globalización han mostrado la importancia y necesidad de reconvertir la ganadería colombiana en sistemas de producción más competitivos, con una visión empresarial a largo plazo y una organización interna proyectada a satisfacer las necesidades de sus clientes. Como respuesta a las demandas de los mercados nacionales e internacionales Colombia ha iniciado un proceso de mejoramiento de los estándares sanitarios en la producción primaria de carne y leche bovina, dando paso a la implementación de buenas prácticas ganaderas (BPG); por parte de la administración municipal de Tauramena, Casanare se realizó el acompañamiento a los productores que estuvieron interesados en el programa, buscando acogerse a las nuevas tecnologías para producir carne y leche de excelente calidad protegiendo la salud de los consumidores, los trabajadores y los animales. El trabajo se realizó con la colaboración del Instituto de Fomento Agroempresarial de Tauramena (IFATA), dentro del marco del cumplimiento de la asistencia técnica pecuaria prestada por parte de la alcaldía municipal a los pequeños y medianos productores a quienes se les brinda la oportunidad de participar en actividades como: asistencia técnica, caracterización de predios, lista de chequeo, planes de mejora y sanitarios, rutas de aprendizaje, gira ganadera interna, escuela de campo agropecuarias (ECAS) y práctica de transformación de subproductos, se consolidó que los productores de carne son 387, quienes manejan razas cebuínas, la cantidad de predios dedicados a la producción lechera son 54 donde se utilizan las razas Girolando, Simmenthal y Pardo Suizo y el sistema doble propósito cuenta con un total de 170 que realizan 
esta tarea con animales de cruces de cebú, Holstein y Pardo Suizo. Se pudo concluir que la caracterización de los predios es una de las metas más importantes para la administración municipal puesto que la actividad permite conocer el estado actual del sector pecuario, y que además actualizaciones deben realizarse constantemente debido a la fluctuación de la actividad ganadera; en cuanto a los productores que hicieron parte del programa de BPG comprendieron que el mismo busca garantizar el bienestar de los animales y la producción inocua de los alimentos.

Palabras clave: Ganadería, bovinos, inocuidad, sanidad, alimentos.

\section{ABSTRACT}

The demands of globalization have shown the importance and necessity of reconvert the Colombian livestock in more competitive production systems, with a long-term business vision and an internal organization designed to meet the needs of its customers. In response to the demands of national and international markets Colombia has initiated a process of improvement of health standards in the primary production of meat and bovine milk, giving way to the implementation of good livestock practices (GMP) by the municipal administration of Tauramena, Casanare the accompaniment was performed to producers who were interested in the program, seeking to take advantage of new technologies to produce meat and milk of excellent quality protecting the health of consumers, workers and animals. The work was done in collaboration with the Institute for Development Agribusiness of Tauramena (IFATA) within the framework of compliance of livestock technical assistance provided by the municipal government to small and medium producers to whom it is given the opportunity to participate in activities such as: technical assistance, characterization of buildings, checklist, improvement plans and health, learning paths, domestic livestock tour, agricultural field school (ECAS), and byproduct processing practice, it was confirmed that meat producers are 387 who handle zebu breeds, the amount of land dedicated to milk production is 54 where races Girolando, Simmenthal and Swiss Brown are used and the dual purpose system has a total of 170 who perform this task with animals of Zebu, Holstein and 
Swiss brown crosses. It was possible to conclude that the characterization of the properties is one of the most important goals for municipal administration since the activity allows know the current state of the livestock sector and that updates must also be done constantly due to the fluctuation of livestock activity; as regards the producers who were part of the GPM program understood that it seeks to ensure the welfare of animals and the safe production of food.

Keywords: Livestock, cattle, safety, health, food.

\section{RESUMO}

As exigências da globalização têm mostrado a importância ea necessidade de reconverter o produção de gado em Colômbia aos sistemas de produção mais competitivos, com uma visão de negócio a longo prazo e uma organização interna projetada para atender às necessidades de seus clientes. Em resposta às exigências dos mercados nacionais e internacionais Colômbia tem iniciou um proceso de melhoria dos padrões de saúde na produção primária de carne e leite bovina, levando à implementação de boas práticas agrícolas (BPG) por parte da administração municipal de Tauramena, Casanare o acompanhamento foi realizado aos produtores que estavam interessados no programa, buscando beneficiar das novas tecnologías para produzir carne e leite de excelente qualidade protegendo a saúde dos consumidores, trabalhadores e animais. $O$ trabalho foi feito em colaboração com o Instituto da promoção do agronegócio de Tauramena (IFATA) no quadro do cumprimento de a assistência técnica pecuária fornecida pela administração municipal aos pequenos e médios produtores a quem thes confere a possibilidade de participar em atividades como: assistência técnica, a caracterização de terra, lista de verificação, planos de melhoria e saúde, caminhos de aprendizagem, passeio doméstica de gado, escola campo agrícola (ECAS) e práticas de processamento do subprodutos, se consolidou que os produtores de carne são 387 quem manejan raças zebuínas, a quantidade de azendas destinados a produção de leite são 54 onde as raças Girolando, Simmenthal e Marrom Suíço são utilizados eo sistema de duplo propósito tem um total de 170 que executar esta tarefa com os animais cruzes Zebu, Holstein e 
Marrom Suíço. Foi concluído que a caracterização das propiedades é uma das metas mais importantes para a administração municipal posto que a actividade permite conhecer o estado actual do sector pecuário, e também actualização constante deve ser realizada devido à flutuação do actvidade pecuária; sobre os produtores que faziam parte do programa BPG entenderam que visa assegurar 0 bem-estar animal e a produção segura de alimentos.

Palavras-chave: Pecuária, gado, segurança, saúde, alimentação.

\section{INTRODUCCIÓN}

La demanda de productos de origen animal en Colombia ha aumentado de acuerdo al índice poblacional y a sus necesidades, razón por la cual se instauró un protocolo que incluye una certificación para las empresas ganaderas que cumplan con los estándares propuestos durante todo el proceso productivo, con el fin de garantizar la calidad e inocuidad del producto final (FEDEGAN, 2010).

Dado que Colombia requiere mejorar el estatus sanitario de los productos que tienen un potencial exportador, para lograr la admisibilidad de la carne, leche y sus productos derivados, se identificó la necesidad de capacitar a los ganaderos para cumplir lo reglamentado por el Instituto Colombiano Agropecuario (ICA) mediante los Decretos 1500 de 2007 (MPS, 2007) y 616 de 2006 (MADR, 2006) y las resoluciones 2341 de 2007 (ICA, 2007) y 3585 de 2008 (ICA, 2008) respectivamente, para optar por la certificación de sus explotaciones.

Las buenas prácticas ganaderas (BPG) son normas que se aplican durante el proceso de producción pecuaria, con el fin de que la empresa ganadera sea sostenible ambiental, económica y socialmente, obteniendo de esta manera productos sanos, seguros y de buena calidad. Las BPG son aplicables a todo lo largo de la cadena productiva bovina: desde el productor o eslabón primario, seguido por el transformador hasta que llegue al consumidor final. Estas normas son aplicables para los diferentes tipos de producción ganadera: lechería, ganado de carne y de doble propósito. El documento CONPES 3676 de julio 19 de 2010 (MADR et al., 2010) tiene como objetivo consolidar la política sanitaria y de 
inocuidad para las cadenas de la leche y carne bovinas; la meta para el año 2015 es aumentar la cobertura de los programas de BPG y trazabilidad en fincas productoras de leche (25\%) y carne (15\%), que proveen a plantas higienizadoras y de beneficio. La implementación de las BPG es un proceso que requiere de tiempo y compromiso para alcanzar las metas propuestas, lo cual será remunerado más adelante ya que se generará: 1) Obtención de productos sanos e inocuos, libres de contaminantes biológicos y químicos; 2) acceso a mercados nacionales e internacionales con mejores precios y oportunidades, disminuyendo la cadena de intermediarios; 3) el manejo de registros proporcionando al productor un mejor conocimiento sobre el comportamiento económico y financiero de su empresa, permitiéndole tomar decisiones administrativas oportunas y apropiadas. 4) Una gestión más próspera en términos productivos y económicos, al mejorar la administración, manejo de insumos, instalaciones y personal, distribución adecuada de labores, aumentando también la competitividad de la empresa al disminuir costos y siendo eficientes; 5) mejoramiento de la imagen de la empresa y sus productos ante los compradores; 6) alternativas para que la comunidad rural mejore las posibilidades de ser incluidos en mercados regionales, nacionales o internacionales; y 7) mejoramiento de las condiciones laborales y sociales del trabajador rural (Uribe et al., 2011).

La certificación de BPG para la finca o empresa ganadera consta de 10 pasos que son verificados y vigilados por el ICA, los cuales son: 1) Inscripción de predios, 2) instalaciones, 3) sanidad animal y bioseguridad, 5) bienestar animal, 6) trazabilidad, 7) buenas prácticas en el uso de medicamentos veterinarios, 8) plan de saneamiento, 9) personal, 10) buenas prácticas alimentación animal y transporte.

En el municipio de Tauramena, Colombia el desarrollo económico se da en gran parte por el sector agropecuario, presentando diferentes sistemas de producción como la agricultura, piscicultura, especies menores (porcinos, ovinos y aves), pesca artesanal y ganadería, siendo esta última predominante por tradición y de gran importancia debido al número de productores que la realizan; con un 
inventario ganadero de más de 1.845 .226 cabezas de ganado en el año 2016 (ICA, 2016); en el 2014 el departamento de Casanare mostró una variación positiva de $3.2 \%$ con respecto al año anterior, y una participación de $8.57 \%$ a nivel nacional (FEDEGAN, 2014). Los sistemas de producción que se manejan en Tauramena son cría, levante, ceba y doble propósito, y la gran mayoría de estos son de tipo extensivo, donde el fuerte es el ganado doble propósito que sale para ceba con un tiempo mínimo de 6 meses para su posterior comercialización en su gran mayoría en la ciudad de Bogotá, y otra parte en ciudades aledañas al municipio.

Para la ejecución de este trabajo, se contó con la colaboración de la Secretaria de Desarrollo Económico y el Instituto de Fomento Agroempresarial de Tauramena (IFATA), entes gestores y promotores de las actividades en pro del desarrollo del sector agropecuario en el municipio; la IFATA es una empresa de gestión económica dotada de personería jurídica, autonomía administrativa y patrimonio propio, que está vinculada a la secretaria de desarrollo económico, la cual recibe su denominación a partir del artículo 01 del acuerdo № 019 del 22 de Septiembre de 2012 (CMT, 2012), cuyo objetivo es fomentar el desarrollo económico y social del municipio a través de la financiación, cofinanciación, ejecución, gerencia y operación de proyectos productivos de impacto para el desarrollo municipal, así como la asesoría y asistencia técnica en los campos de la producción, transformación y comercialización en los diferentes sectores de la economía, como lo son el agropecuario, empresarial, industrial, comercial y turístico, en forma individual o asociada.

\section{METODOLOGÍA}

Tauramena se encuentra localizado en la zona suroccidental del departamento de Casanare (Figura 1), tiene una extensión aproximada de $2607.2 \mathrm{~km}^{2}$ equivalentes al $5.8 \%$ del total del departamento, cuya extensión es de $44640 \mathrm{~km}^{2}$, se ubica en la región natural conocida como Orinoquia colombiana que tiene una extensión de $254445 \mathrm{~km}^{2}$ equivalentes al $22.3 \%$ de la superficie nacional. Su cabecera municipal se localiza a los $5.01^{\prime} 07^{\prime \prime}$ de latitud norte y $72.45^{\prime} 19$ " de longitud oeste, 
latitud de la cabecera municipal (metros sobre el nivel del mar): 460, temperatura media: $25.3^{\circ} \mathrm{C}$ en su parte plana con valores máximos que oscilan entre 33.6 y $39.8^{\circ} \mathrm{C}$ y mínimos que oscilan entre 12 y $19^{\circ} \mathrm{C}$ (Alcaldía de Tauramena, 2016).

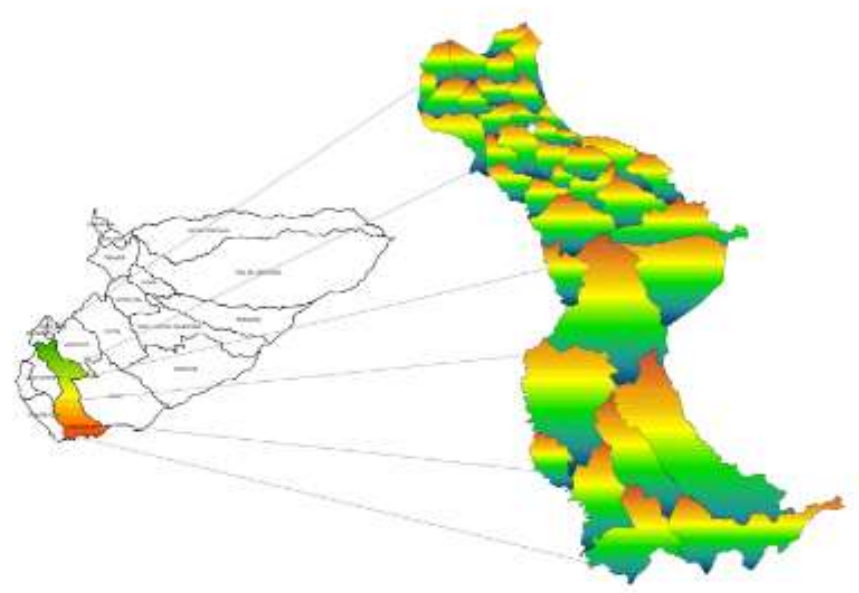

Figura 1. Municipio de Tauramena, Departamento de Casanare, Colombia. Fuente: Alcaldía de Tauramena, (2016)

El presente trabajo se realizó con colaboración del IFATA, dentro del marco del cumplimiento de la asistencia técnica pecuaria prestada por parte de la alcaldía municipal a los pequeños y medianos productores a quienes se les brinda la oportunidad de participar en actividades como: 1) Asistencia técnica que contempla las actividades de asesoramiento pecuario directo a los productores y asesoramiento en BPG; 2) caracterización de predios, es decir realizar la caracterización de las cadenas productivas más relevantes del municipio (ganadería) lo cual permite determinar el estado en el que se encuentran los predios que se pretenden certificar, determinando así lo que falta en cada uno de ellos para el proceso de certificación en BPG; 3) listas de chequeo que permiten determinar lo que está y por lo tanto lo que hace falta en el predio para estar al día con los requisitos establecidos por el ICA; 4) planes de mejora para complementar las actividades basándose en la lista de chequeo, en los cuales se diseñan los protocolos y procesos que se deben llevar acabo en la producción; 5) planes sanitarios que implican diseño, implementación y seguimiento para cada predio donde se describen las actividades sanitarias a realizar y su respectivo cronograma, como esquemas de vacunación y desparasitaciones externas e 
internas; 6) rutas de aprendizaje para la transmisión de conocimientos en tecnología de las cadenas productivas más relevantes, donde se trataron temas técnicos, sanitarios, ambientales y socioeconómicos; 7) gira ganadera interna para la transmisión de conocimientos por parte de los productores que se encuentran dentro del proceso de buenas prácticas en la región; 8) ECAS: escuelas de campo agropecuarias para la transferencia de tecnología en las cadenas productivas en BPG, alimentación animal, producción sostenible, manejo pos-cosecha y valor agregado (Groeneweg et al., 2007; Pezo et al., 2007); 9) práctica de transformación de subproductos para la elaboración de productos de origen lácteo y cárnico, dándole un valor agregado a los mismos.

\section{DIAGNÓSTICO DE LA PRODUCCIÓN PECUARIA EN TAURAMENA}

\section{Elaboración de listas de chequeo}

Para llevar a cabo el diagnóstico de fincas pecuarias en buenas prácticas es necesario diligenciar listas de chequeo, permitiendo determinar las medidas necesarias que se deben implementar para estar al día con los requisitos establecidos por el ente regulador (ICA); las listas de chequeo que se emplean en este proceso están basada en las listas usadas por el ICA con el fin de evaluar, mejorar y cumplir a cabalidad con las exigencias del mismo. Dentro de la evaluación realizada se hizo énfasis en las fincas que serían usadas en el presente trabajo teniendo en cuenta que se encuentran inscritas en el programa de BPG apoyado por la asistencia técnica prestada por la alcaldía municipal en colaboración con el IFATA dentro de las cuales se encuentran alrededor de 45 predios; 10 de los cuales cumplen con un mayor porcentaje de los parámetros requeridos para la certificación. Se encontró que son dos los predios que están en proceso de certificación en producción de leche y cuatro en producción de carne.

Los puntos evaluados por las listas de chequeo se dividen en ítems según el área o concepto a evaluar, a partir de los parámetros calificados en esta lista se elaboran los planes de mejora que se implementaron en cada una de las fincas, buscando en lo posible cumplir a cabalidad con lo establecido y requerido en la 
norma; dentro de los ítems evaluados están: 1) Sanidad animal y bioseguridad, 2) cuarto o tanque de enfriamiento, 3) sistema de ordeño, 4) rutina de ordeño, 5) protección contra la contaminación de la leche, 6) leche, 7) utensilios y equipos de del ordeño, 8) suministro y calidad de agua, 9) control de medicamentos veterinarios e insumos agropecuarios, 10) saneamiento y control de plagas, 11) instalaciones y otras áreas, 12) registros y documentación, 13) bienestar animal y 14) personal.

\section{Caracterización de predios ganaderos}

En el municipio de Tauramena se identifican varios renglones productivos del sector agropecuario, destacándose la explotación bovina (Figura 2) como la actividad más importante en generación de ingresos, también encadenamientos con sectores denominados prioritarios como cacao, palma, especies menores (aves de corral, cerdos y otros), frutales como piña, cítricos, y sectores con alto potencial de desarrollo que se denominan tradicionales y promisorios donde se destaca la producción tecnificada de plátano.

Respecto al establecimiento de actividades de ganadería en sus diferentes etapas (cría, levante, ceba o doble propósito), existía según reporte de FEDEGAN para el mes de Mayo de 2013, en total 1177 predios dedicados a la producción bovina con 152.071 cabezas de ganado, distribuidas, de manera uniforme, principalmente en las veredas: Agua blanca, Yaguaros, Jagüito, Chitamena, Visinaca, Corocito, Piñalito, Cabañas, lquia, El Guira, La Urama, Vigía, Delicias, Villa rosa, Palmar, Aguamaco, Batallera, Aceite alto, Guafal del caja, Chaparral, La lucha, Lagunitas, Cuernavaca, Monserrate, Tunupe, Juve, Raizal, Carupana, Güichire, Oso, La esmeralda, Zambo, Bendiciones y Paso cusiana (FEDEGAN, 2014).

La identificación y caracterización de las cadenas productivas más relevantes, entre ellas la ganadería, permite determinar el estado en el que se encuentran los predios que se pretenden certificar, determinando así lo existente y lo que falta en cada uno de ellos para el proceso de implementación de BPG. Con el fin de facilitar la planificación y el conocimiento de la actividad agropecuaria, la alcaldía 
municipal diseñó desde hace varios años el sistema de información agropecuario (SIATA), herramienta basada en las Tecnologías de la información y la comunicación (TIC) que cuenta con la facilidad del ingreso de datos y posibilidad de acceso masivo mediante internet, las cuales fueron construidas colectivamente para fortalecer los procesos de planeación y desarrollo agropecuario de Tauramena.
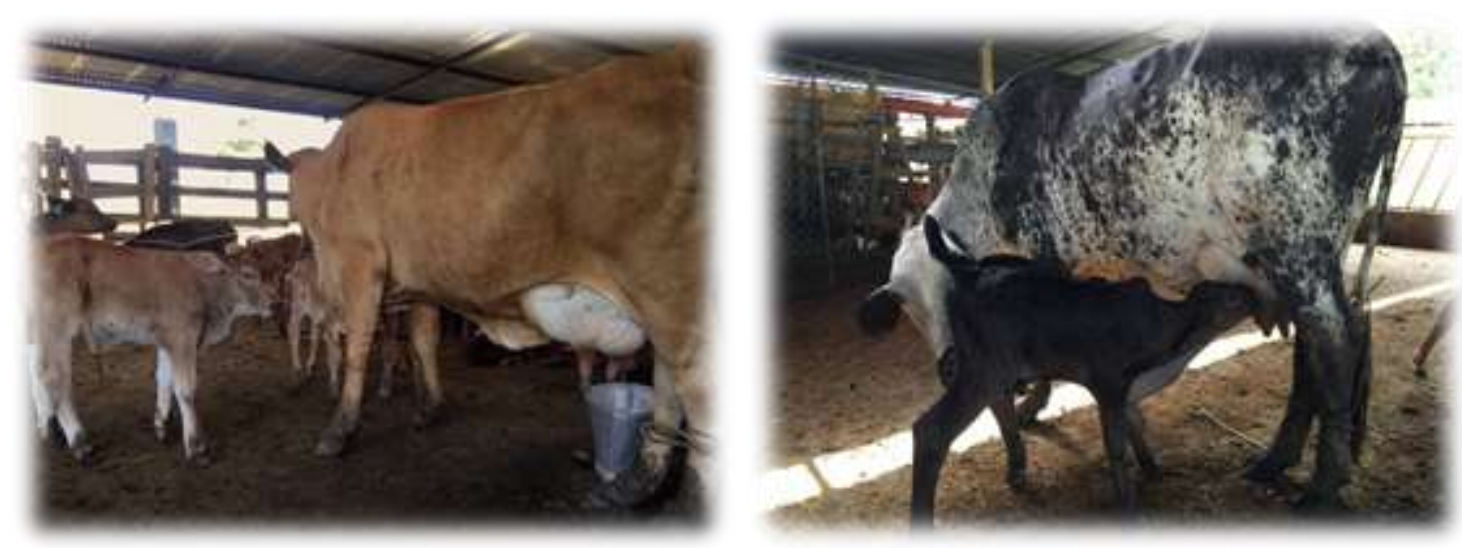

Figura 2. Predios dedicados a la producción de leche Fuente: Fotografía cortesía de Zambrano, MVZ, Unillanos.

\section{Registro de usuarios asistencia técnica (RUAT) consolidados en el SIATA en Tauramena}

De acuerdo a esta información, el sistema de producción bovina es un renglón económico importante en éste municipio, arrojando datos de aproximadamente 1717 granjas ganaderas registradas en censos agropecuarios, donde 1246 se encuentran dedicadas a la producción doble propósito empleando razas y cruces de Cebú, Holstein y Pardo Suizo seguido por la producción de carne, y finalmente la producción láctea que se ha venido reduciendo sustancialmente debido a las fluctuaciones en el costo de la comercialización de la leche.

Se consolidó que los productores de carne (Tabla 1) son 387 de un total de 397 predios donde las veredas que presentan mayor producción son: Visinaca, Delicias y Yaguaros, donde se maneja las razas cebuínas aprovechando sus características de adaptabilidad, rusticidad y habilidad materna. El sistema doble propósito (Tabla 2), cuenta con un total de 170 productores de los 173 predios 
encuestados, siendo las veredas Delicias, Aceite alto y Agua blanca las de mayor número de predios que realizan esta labor. La cantidad de predios que llevan a cabo la labor de producción lechera del municipio son 54 (Tabla 3), siendo la vereda Aguamaco la de mayor número de fincas (12) dedicadas a ésta actividad, donde se utilizan las razas Girolando, Simmenthal y Pardo Suizo.

Tabla 1. Sistema de producción bovina tipo carne

\begin{tabular}{|c|c|c|c|c|}
\hline \multirow{2}{*}{$\begin{array}{c}\text { Vereda } \\
\text { Aceite Alto }\end{array}$} & \multicolumn{2}{|c|}{ No. Predios } & \multicolumn{2}{|c|}{ No. Productores } \\
\hline & 3 & $0.8 \%$ & 3 & $0.8 \%$ \\
\hline Agua Blanca & 15 & $3.9 \%$ & 15 & $4.0 \%$ \\
\hline Aguamaco & 16 & $4.1 \%$ & 16 & $4.3 \%$ \\
\hline Batallera & 16 & $4.1 \%$ & 16 & $4.3 \%$ \\
\hline Bendiciones & 9 & $2.3 \%$ & 6 & $1.6 \%$ \\
\hline Cabañas & 1 & $0.3 \%$ & 1 & $0.3 \%$ \\
\hline Carupana & 15 & $3.9 \%$ & 15 & $4.0 \%$ \\
\hline Chaparral & 16 & $4.1 \%$ & 16 & $4.3 \%$ \\
\hline Chitamena & 16 & $4.1 \%$ & 16 & $4.3 \%$ \\
\hline Corocito & 12 & $3.1 \%$ & 12 & $3.2 \%$ \\
\hline Cuernavaca & 11 & $2.8 \%$ & 11 & $2.9 \%$ \\
\hline Delicias & 33 & $8.5 \%$ & 33 & $8.8 \%$ \\
\hline El Guira & 3 & $0.8 \%$ & 3 & $0.8 \%$ \\
\hline Guafal del Caja & 5 & $1.3 \%$ & 5 & $1.3 \%$ \\
\hline Guichire & 8 & $2.1 \%$ & 6 & $1.6 \%$ \\
\hline Iquia & 23 & $5.9 \%$ & 23 & $6.1 \%$ \\
\hline Jaguito & 4 & $1.0 \%$ & 4 & $1.1 \%$ \\
\hline Juve & 3 & $0.8 \%$ & 3 & $0.8 \%$ \\
\hline La Esmeralda & 1 & $0.3 \%$ & 1 & $0.3 \%$ \\
\hline La Urama & 25 & $6.5 \%$ & 25 & $6.7 \%$ \\
\hline Lagunitas & 5 & $1.3 \%$ & 5 & $1.3 \%$ \\
\hline Monserrate & 8 & $2.1 \%$ & 8 & $2.1 \%$ \\
\hline Oso & 8 & $2.1 \%$ & 7 & $1.9 \%$ \\
\hline Palmar & 4 & $1.0 \%$ & 4 & $1.1 \%$ \\
\hline Paso Cusiana & 1 & $0.3 \%$ & 1 & $0.3 \%$ \\
\hline Tunupe & 12 & $3.1 \%$ & 12 & $3.2 \%$ \\
\hline Vigia & 20 & $5.2 \%$ & 20 & $5.3 \%$ \\
\hline Villarosa & 19 & $4.9 \%$ & 19 & $5.1 \%$ \\
\hline Visinaca & 36 & $9.3 \%$ & 32 & $8.5 \%$ \\
\hline Yaguaros & 36 & $9.3 \%$ & 36 & $9.6 \%$ \\
\hline Zambo & 3 & $0.8 \%$ & 3 & $0.8 \%$ \\
\hline
\end{tabular}


Tabla 2. Sistema de producción bovina doble propósito

\begin{tabular}{|c|c|c|c|c|}
\hline \multirow{2}{*}{$\begin{array}{c}\text { Vereda } \\
\text { Aceite Alto }\end{array}$} & \multicolumn{2}{|c|}{ No. Predios } & \multicolumn{2}{|c|}{ No. Productores } \\
\hline & 23 & $13.3 \%$ & 23 & $13.5 \%$ \\
\hline Agua Blanca & 29 & $16.8 \%$ & 29 & $17.1 \%$ \\
\hline Aguamaco & 4 & $2.3 \%$ & 4 & $2.4 \%$ \\
\hline Batallera & 1 & $0.6 \%$ & 1 & $0.6 \%$ \\
\hline Bendiciones & 2 & $1.2 \%$ & 2 & $1.2 \%$ \\
\hline Cabañas & 4 & $2.3 \%$ & 4 & $2.4 \%$ \\
\hline Carupana & 12 & $6.9 \%$ & 12 & $7.1 \%$ \\
\hline Chaparral & 5 & $2.9 \%$ & 5 & $2.9 \%$ \\
\hline Chitamena & 2 & $1.2 \%$ & 2 & $1.2 \%$ \\
\hline Corocito & 12 & $6.9 \%$ & 12 & $7.1 \%$ \\
\hline Cuernavaca & 2 & $1.2 \%$ & 2 & $1.2 \%$ \\
\hline Delicias & 22 & $12.7 \%$ & 22 & $12.9 \%$ \\
\hline El Guira & 2 & $1.2 \%$ & 2 & $1.2 \%$ \\
\hline Iquia & 2 & $1.2 \%$ & 2 & $1.2 \%$ \\
\hline Jaguito & 12 & $6.9 \%$ & 10 & $5.9 \%$ \\
\hline Juve & 2 & $1.2 \%$ & 2 & $1.2 \%$ \\
\hline La Esmeralda & 4 & $2.3 \%$ & 4 & $2.4 \%$ \\
\hline La Lucha & 1 & $0.6 \%$ & 1 & $0.6 \%$ \\
\hline Lagunitas & 2 & $1.2 \%$ & 1 & $0.6 \%$ \\
\hline Monserrate & 1 & $0.6 \%$ & 1 & $0.6 \%$ \\
\hline Oso & 4 & $2.3 \%$ & 4 & $2.4 \%$ \\
\hline Palmar & 8 & $4.6 \%$ & 8 & $4.7 \%$ \\
\hline Raizal & 2 & $1.2 \%$ & 2 & $1.2 \%$ \\
\hline Villarosa & 2 & $1.2 \%$ & 2 & $1.2 \%$ \\
\hline Visinaca & 4 & $2.3 \%$ & 4 & $2.4 \%$ \\
\hline Yaguaros & 7 & $4.0 \%$ & 7 & $4.1 \%$ \\
\hline Zambo & 2 & $1.2 \%$ & 2 & $1.2 \%$ \\
\hline
\end{tabular}


Tabla 3. Sistema de producción bovina tipo leche

\begin{tabular}{ccccc}
\hline Vereda & \multicolumn{2}{c}{ No. Predios } & \multicolumn{2}{c}{ No. Productores } \\
\hline Aceite Alto & 1 & $1.9 \%$ & 1 & $1.9 \%$ \\
Agua Blanca & 2 & $3.7 \%$ & 2 & $3.7 \%$ \\
Aguamaco & 12 & $22.2 \%$ & 12 & $22.2 \%$ \\
Bendiciones & 1 & $1.9 \%$ & 1 & $1.9 \%$ \\
Chaparral & 3 & $5.6 \%$ & 3 & $5.6 \%$ \\
Cuernavaca & 1 & $1.9 \%$ & 1 & $1.9 \%$ \\
Delicias & 2 & $3.7 \%$ & 2 & $3.7 \%$ \\
Guafal del Caja & 4 & $7.4 \%$ & 4 & $7.4 \%$ \\
Iquia & 2 & $3.7 \%$ & 2 & $3.7 \%$ \\
Jaguito & 1 & $1.9 \%$ & 1 & $1.9 \%$ \\
Juve & 1 & $1.9 \%$ & 1 & $1.9 \%$ \\
Lagunitas & 2 & $3.7 \%$ & 2 & $3.7 \%$ \\
Monserrate & 1 & $1.9 \%$ & 1 & $1.9 \%$ \\
Oso & 8 & $14.8 \%$ & 8 & $14.8 \%$ \\
Palmar & 7 & $13.0 \%$ & 7 & $13.0 \%$ \\
Paso Cusiana & 1 & $1.9 \%$ & 1 & $1.9 \%$ \\
Visinaca & 2 & $3.7 \%$ & 2 & $3.7 \%$ \\
Yaguaros & 3 & $5.6 \%$ & 3 & $5.6 \%$ \\
\hline
\end{tabular}

\section{ASISTENCIA TÉCNICA}

La extensión rural es uno de los medios de transmisión del conocimiento para la transferencia de tecnología en las cadenas productivas, donde se tratan temas técnicos, sanitarios, ambientales y socioeconómicos. Para brindar la asistencia técnica en este caso se trabajó directamente con los productores asesorándolos en BPG en diferentes veredas, teniendo en cuenta la demanda de los productores, además se atendieron diferentes casos clínicos y productivos (120) según las necesidades y casuística reportadas por los ganaderos que se asistieron, encontrando bovinos con mastitis, partos distócicos, retenciones de placenta, hernias abdominales y umbilicales, papilomatosis, claudicaciones, abscesos, escoriaciones, anemia, hipertermia, deshidratación, y también se realizó 
castración de cerdos, captura de hematófagos, vacunación y desparasitación de bovinos y equinos.

La atención de los casos clínicos y su respectivo seguimiento se llevó a cabo por parte del profesional a cargo, pasante de Medicina Veterinaria y Zootecnia y el técnico pecuario. A partir del consolidado de los casos atendidos se puede concluir que lo más frecuente correspondió a mastitis (aguda o crónica), partos distócicos, y retenciones de placenta; el abordaje médico que se dio a cada caso clínico fue sintomático, haciendo uso de productos comerciales como en mastitis antibióticos intramamarios secantes, y reforzando en casos severos con antibiótico sistémico; cuando se presentó retención de placenta, se procedió a la palpación vía transrectal y vaciamiento del contenido del útero, usando una cefalosporina para el tratamiento de endometritis; y para los casos de distocia se realizó palpación transvaginal para determinar la posición del feto y así realizar su extracción, se garantizó la atención del neonato y el consumo del calostro.

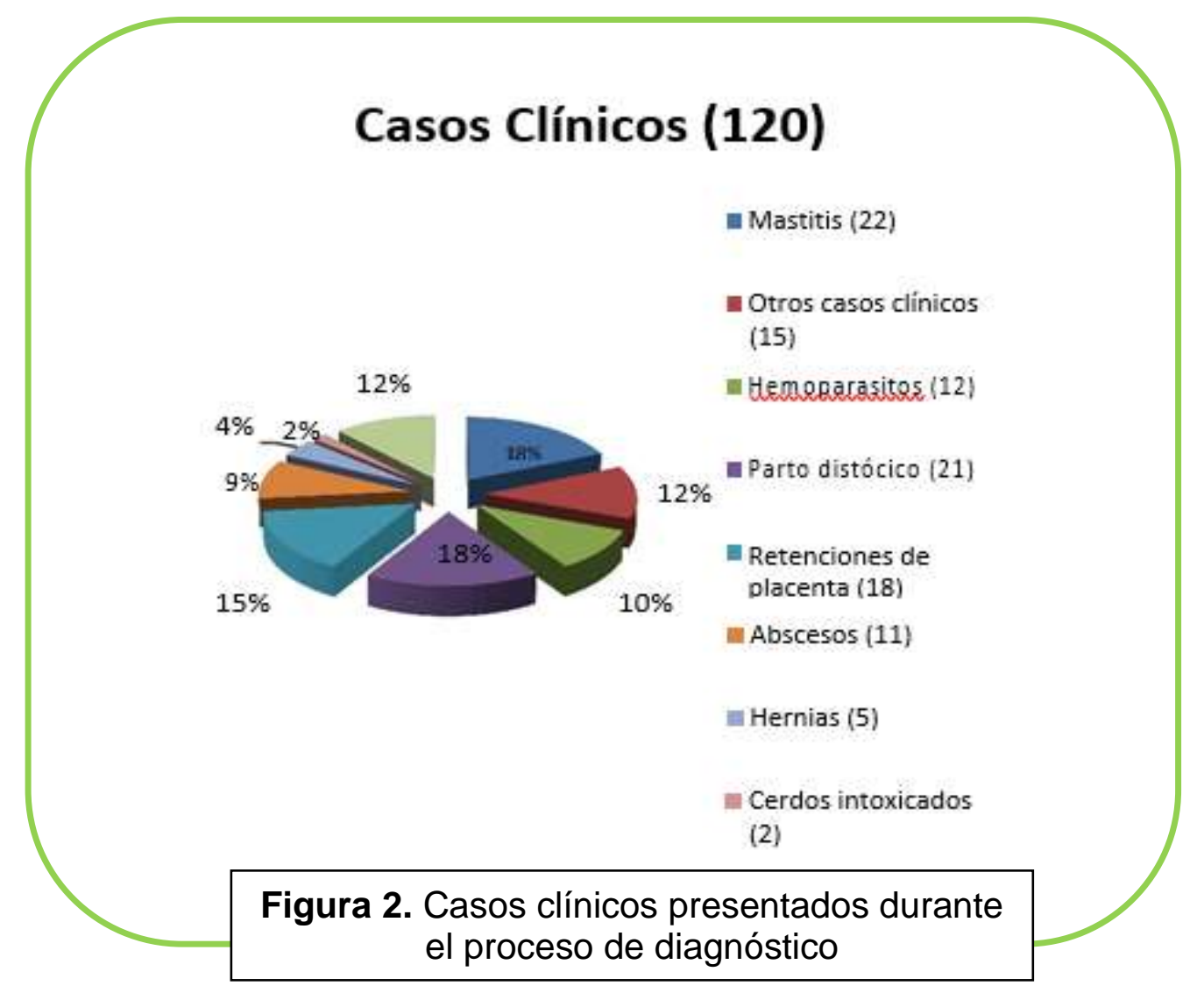




\section{DISEÑO DE PLANES DE MEJORA}

El plan de mejora se realizó para complementar las actividades de asistencia, y se basó en la lista de chequeo, el cual fue construido entre el productor y el asistente técnico, e incluyó el diseño de protocolos y procedimientos que se deben llevar acabo en la producción para cumplir con lo establecido por el ente regulador. Después de realizar los diagnósticos se encontraron predios con implementación de BPG adelantados en cuanto a procesos de manejo e instalaciones, así como productores que estaban iniciando dentro del programa de BPG, con los cuales se procedió a implementar y realizar los diferentes procesos.

\section{Diseño e implementación, socialización y seguimiento de plan sanitario}

Se diseñó un plan de actividades sanitarias con cronogramas de vacunación y desparasitaciones tanto externas como internas, puesto que las fincas destinadas a la producción de alimentos para el consumo humano (leche y/o carne) deben contar con un plan sanitario documentado, que incluya la prevención, diagnóstico y manejo de enfermedades comunes (endémicas) y el manejo de las enfermedades de control oficial (fiebre aftosa, brucelosis, rabia, tuberculosis y las que el ICA determine), así como prácticas de manejo, preventivas o curativas.

\section{Procedencia de animales}

Los semovientes deben estar sanos y provenir de fincas registradas ante el ICA, con la documentación que acredite el cumplimiento de todos los requisitos sanitarios, previniendo de esta manera el ingreso de enfermedades (Garzón y Nieto, 2011).

\section{Postulación}

Los productores de leche que deseen participar para la obtención de la certificación como hato libre de brucelosis y tuberculosis bovina, deben contar con la certificación oficial vigente en BPG, y deben cumplir con las medidas sanitarias y directrices de los programas oficiales del ICA para el control y erradicación de 
brucelosis y tuberculosis, puesto que dichas enfermedades representan un grave riesgo para la salud de los consumidores y una limitación en la comercialización de la leche y sus derivados.

\section{Control oficial}

Los programas de vacunación contra enfermedades oficiales se deben cumplir teniendo en cuenta los ciclos establecidos por el ICA para fiebre aftosa y brucelosis y mantener el registro único de vacunación (RUV) en el predio; es necesario vacunar y mantener el registro de las enfermedades propias de la región y del sistema productivo, por ejemplo: clostridiales y aquellas que el ICA considere pertinentes; además los predios deben tener un área de cuarentena destinada a la observación y adaptación de los animales que ingresan al predio.

\section{Área de cuarentena}

Debe estar ubicada de manera tal que no constituya un riesgo sanitario para otros animales de la finca, donde los animales nuevos deben permanecer 21 días y los enfermos deben ser identificados para brindar el tratamiento correspondiente.

\section{Plan de atención de emergencia}

Ante cualquier sospecha de enfermedad con síntomas compatibles con aftosa o rabia o la presencia de mortalidad inusual, se debe avisar de inmediato a la oficina más cercana del ICA; además es necesario contar con un instructivo fijado en un lugar visible, donde estén los nombres de las personas con sus respectivos números de teléfono, que deben ser notificadas en las fincas y el personal del ICA que debe ser informado.

\section{Registro de ingreso y salida de vehículos y visitantes}

Debe incluir la fecha, el nombre del visitante, la placa del vehículo, su procedencia, la actividad a realizar y el teléfono de contacto. Como complemento a dichos planes de mejora (Cuadro 1) se realizaron distintas actividades en las que se consideraron entre otros temas: buenas prácticas de ordeño, cuidado del 
recién nacido, elaboración de ensilajes y bloques multinuticionales, manejo de enfermedades y planes sanitarios; las capacitaciones otorgadas a los ganaderos se realizaron para afianzar sus conocimientos, lo cual se complementó organizando charlas y giras donde se trataron diferentes temas de interés en predios de productores que se acogieron al programa, y se les realizo el apoyo técnico para cumplir los requerimientos para ser certificados en BPG.

Cuadro 1. Capacitaciones para ganaderos en Tauramena

\begin{tabular}{|c|c|c|}
\hline Nombre curso & Objetivo & Actividades \\
\hline $\begin{array}{l}\text { Buenas prácticas } \\
\text { ganaderas al } \\
\text { ordeño }\end{array}$ & $\begin{array}{c}\text { Transmitir conocimientos } \\
\text { teórico-prácticos sobre } \\
\text { buenas prácticas ganaderas } \\
\text { al ordeño }\end{array}$ & $\begin{array}{l}\text { Se hace el ordeño, realizando el test } \\
\text { de mastitis, dando una explicación } \\
\text { teórica del procedimiento de ordeño } \\
\text { según normatividad del ICA, } \\
\text { llevando a cabo la actividad } \\
\text { recreativa "la vaca lechera" }\end{array}$ \\
\hline $\begin{array}{c}\text { Cuidados en el } \\
\text { parto y perinatales }\end{array}$ & $\begin{array}{l}\text { Transferir conocimientos } \\
\text { sobre signología del parto, } \\
\text { cuidados y atención de la } \\
\text { cría y la vaca. }\end{array}$ & $\begin{array}{l}\text { Capacitación ilustrada del cuidado } \\
\text { en el parto y postparto, cuándo y } \\
\text { cómo asistir a la vaca, evaluación de } \\
\text { la posición del ternero en el canal de } \\
\text { parto, estimulación de la respiración, } \\
\text { manejo del calostro, limpieza y } \\
\text { desinfección del cordón umbilical y la } \\
\text { alimentación del neonato. Se } \\
\text { entregaron folletos para que el } \\
\text { productor afianzara por medio de la } \\
\text { lectura sus conocimientos. }\end{array}$ \\
\hline $\begin{array}{l}\text { Elaboración de } \\
\text { bloques y ensilaje }\end{array}$ & $\begin{array}{l}\text { Transmitir conocimientos } \\
\text { sobre suplementación } \\
\text { alimenticia en bovinos } \\
\text { mediante la elaboración de } \\
\text { bloques multinutrionales. }\end{array}$ & $\begin{array}{l}\text { Capacitación sobre nutrición en } \\
\text { bovinos, sus requerimientos, } \\
\text { formulación y elaboración de } \\
\text { bloques multinutricionales a base de } \\
\text { harina (tulipán), melaza, urea, cal, } \\
\text { sal mineraliza, y preparación de silo } \\
\text { con pasto de corte, por parte de los } \\
\text { ganaderos en compañía del equipo } \\
\text { técnico. }\end{array}$ \\
\hline $\begin{array}{l}\text { Tratamiento de } \\
\text { casos clínicos }\end{array}$ & $\begin{array}{l}\text { Dar a conocer las causas, } \\
\text { diagnóstico y tratamiento de } \\
\text { la diarrea en terneros }\end{array}$ & $\begin{array}{l}\text { Conferencia en la cual se enseñó a } \\
\text { calcular el porcentaje de } \\
\text { deshidratación y las técnicas de para } \\
\text { rehidratar. Se habló acerca de la } \\
\text { incidencia de la fiebre, mastitis } \\
\text { clínica y edema mamario de las } \\
\text { novillas. Se realizó el ejercicio } \\
\text { práctico de inyectología en bovinos, } \\
\text { equinos y caninos, administración de }\end{array}$ \\
\hline
\end{tabular}




\section{Enfermedades parasitarias}

Gira ganadera interna

Escuela de Campo Agropecuaria (ECA)

Transformación de subproductos
Conocer las principales enfermedades parasitarias que afectan a los bovinos y su impacto en la economía ganadera.

Enseñar sobre el manejo adecuado de medicamentos.

Entregar certificados a quienes participaron en el curso de ganadería y buenas prácticas.

Visualizar modelos productivos certificados dentro del municipio, creando retroalimentación con experiencias para incentivar a los productores a certificar sus predios en BPG.

Capacitar por medio de actividades pedagógicas y prácticas sobre el manejo de ganadería: alimentación, buenas prácticas de manejo de medicamentos, trasplante de embriones y medios virtuales de comunicación.

Enseñar a los productores procedimientos que generan valor agregado a su producto primario. medicamentos por vía intravenosa, subcutánea e intramuscular.

Capacitación sobre estrategias de prevención y control de enfermedades parasitarias. Se enseñó la ejecución de planes sanitarios en las fincas, mediante el uso de cronogramas sanitarios.

Gira técnica pedagógica a la cual asistieron productores interesados en la implementación de las BPG.

Capacitación sobre la transferencia de tecnología en las cadenas productivas en BPG, BPP, alimentación animal, y producción sostenible, con el apoyo de entidades como laboratorios Provet, SOMEX, ANUNCIALO, IFATA, ASOSIMMENTAL, y la agropecuaria la Nueva Florida.

Procesos de transformación de leche en queso siete cueros y doble crema, yogurt de melocotón y fresa.

EI IFATA se hizo responsable de esta actividad junto con la participación activa de los asistentes.

\section{CONSIDERACIONES FINALES}

La caracterización de los predios en el municipio es una de las metas más importantes para la administración municipal, actividad que se lleva a cabo permitiendo conocer el estado actual del sector pecuario, además actualizaciones deben realizarse constantemente debido a la fluctuación de la actividad ganadera; como se puedo evidenciar en la producción y comercialización de la leche que se 
ve afectada por la caída sustancial en el precio de venta, donde inicialmente según los productores el pago se encontraba entre los $\$ 750$ y $\$ 800$ por litro, hasta alcanzar luego un descenso gradual a $\$ 500$, razón por la cual los productores justifican el cambio de la actividad productiva o la transformación del producto obtenido. Por otro lado, la comercialización de la carne se ha mantenido estable en promedio $\$ 2900$ peso vivo, en la planta de beneficio del municipio. Se llevó a cabo el seguimiento a tres predios certificados los cuales solo requirieron la actualización del certificado médico del personal y la actualización de registros.

Las fincas que se encontraban en proceso de certificación iniciaron la implementación de los requisitos fundamentales, y en la actualidad se encuentran a la espera de la visita por parte del personal del ICA para la inspección y posterior registro del predio en el programa BPG. Los planes de mejora se realizaron a partir de la elaboración de las listas de chequeo junto con el propietario de cada finca, posterior a un recorrido de verificación del estado y porcentaje de cumplimiento de los requisitos; junto con los productores que hacen parte del programa de BPG se pudo evidenciar que se cumplía a cabalidad con los requisitos, en el caso de los predios en proceso de certificación los puntos evaluados fueron los planes sanitarios animales buscando garantizar el bienestar de los mismos y la producción inocua de los subproductos.

\section{CONCLUSIONES}

La capacitación de los productores fue una de las actividades desarrolladas durante la práctica profesional donde se transmitió a los ganaderos temas de interés dando respuesta a dudas e inquietudes que ellos manifestaron, y se realizaron capacitaciones para el abordaje básico de los animales y la transformación de materias primas, prestando asistencia técnica pecuaria de forma generalizada a los medianos y pequeños productores del municipio, donde fue posible evidenciar la presentación frecuente de casos de mastitis, retenciones de placenta y partos distócicos, el abordaje y seguimiento oportuno de éstos permitió que el tratamiento fuera efectivo en la totalidad de los casos. 
La pasantía profesional es la culminación de una etapa de desarrollo y preparación profesional, que su ejecución se pone en práctica los conocimientos adquiridos a lo largo del pregrado en medicina veterinaria y zootecnia, específicamente en el área de sanidad en la producción, así como la adquisición de nuevos conocimientos gracias al acercamiento y acompañamiento de los productores quienes de acuerdo a su experiencia en el campo retroalimentan de manera positiva a los profesionales.

\section{REFERENCIAS BIBLIOGRÁFICAS}

1. Alcaldía Tauramena-Casanare. Acuerdo N. 019 del 22 de Septiembre de 2012. Reforma del fondo de fomento agropecuario de Tauramena. Entidades descentralizadas. Recuperado 14 Abril 2016. Disponible En: http://www.tauramenacasanare.gov.co/Entidades descentralizadas.shtml?apc=lbEmpresas\%20de\% 20econom\%EDa\%20mixta-1-\&x=2274497

2. Alcaldía de Tauramena-Casanare. Información General del Municipio. 2016. Recuperado 02 Mayo 2016. Disponible En: http://www.tauramenacasanare.gov.co/informacion general.shtml\#geografia

3. Alcaldía de Tauramena-Casanare. Mapas geográficos del municipio. 2016. Recuperado 02 Mayo 2016. Disponible En: http://www.tauramenacasanare.gov.co/mapas municipio.shtml?apc=bcxx-1-\&x=2625714

4. Alcaldía Municipal de Tauramena, Secretaría de Desarrollo Económico. Ganadería de carne. 2016. Recuperado 02 Mayo 2016. Disponible En: http://www.siata.com.co/sistemas/sistemasp.php?id=24

5. Alcaldía Municipal de Tauramena, Secretaría de Desarrollo Económico. Ganadería de leche. 2016. Recuperado 02 Mayo 2016. Disponible En: http://www.siata.com.co/sistemas/sistemasp.php?id=23

6. Alcaldía Municipal de Tauramena - Secretaría de desarrollo económico. Ganadería doble propósito. 2016. Recuperado 02 Mayo 2016. Disponible En: http://www.siata.com.co/sistemas/sistemasp.php?id=25

7. CMT, Concejo Municipal Tauramena. Acuerdo Municipal N. 019 de 2012. Reforma del Fondo de Fomento Agropecuario de Tauramena. 2012. Recuperado 21 Septiembre 2015. Disponible En: http://tauramenacasanare.gov.co/apc-aafiles/32313835323737646332303666366238/acuerdo-019-de-22-de-sept-de2012.pdf

8. CMT, Concejo Municipal Tauramena. Acuerdo Municipal N. 001 de 2014. Esquema de ordenamiento territorial para el municipio de Tauramena, Casanare. 2014. Recuperado 21 Septiembre 2015. Disponible En: http://www.concejo-tauramena-casanare.gov.co/apc-aafiles/653432613161303133333386538346461/acuerdo-001-e.o.t-tauramena.pdf 
9. Contreras JC. Actualización y caracterización de sectores productivos del municipio de Tauramena, Casanare. Tauramena, Casanare, Colombia. IFATA. Contrato Interadministrativo N. 128 de 2013. 2014. Recuperado 02 Mayo 2016.

Disponible

En: http://www.redlactea.org/documentos/manual\%20bpg\%20colombia.pdf

10. Federación Colombiana de Ganaderos (FEDEGAN). Carta Fedegan N. 120: País ganadero y exportador. 2010. Recuperado 21 Septiembre 2015. Disponible En: http://www.fedegan.org.co/carta-fedegan-120-pais-ganadero-yexportador

11. Federación Colombiana de Ganaderos (FEDEGAN). Buenas prácticas ganaderas. 2013. Recuperado 02 Mayo 2016. Disponible En: http://www.fedegan.org.co/programas/buenas-practicas-ganaderas

12. FEDEGAN, Federación Colombiana de Ganaderos, Estadísticas Inventario bovino nacional. 2014. Recuperado 18 Octubre 2015. Disponible En: http://www.fedegan.org.co/estadisticas/inventario-bovino-nacional

13. Garzón MA, Acosta JM. Bienestar animal: Nuevo reto para la ganadería. Guía metodológica. Instituto Colombiano Agropecuario (ICA). Ed Produmedios. Bogotá, Colombia. 19 p. 2006.

14. Garzón, MA, Nieto A. Las buenas prácticas ganaderas en la producción de leche. Ed Produmedios, Bogotá, Colombia Instituto Colombiano Agropecuario (ICA). 32 p. 2011.

15. Gobernación de Arauca, Secretaria de Desarrollo Agropecuario y Sostenible Departamental, Cartilla para la aplicación de las buenas prácticas ganaderas (BPG'S), Sistemas silvopastoriles y conservación de forrajes. Arauca, Colombia, 42 p. 2011. Recuperado 02 Mayo 2016. Disponible En: http://www.fundaset.org.co/wpcontent/uploads/descargables/cartillas\%20BPG.pdf

16. Groeneweg K, Buyu G, Romney D, Minjauw B. Escuelas de campo para productores pecuarios: Normas para la facilitación y manual técnico. International Livestock Research Institute, Nairobi, Kenya. 252 p. 2007.

17. Federación Colombiana de Ganaderos (FEDEGAN), Técnicas Ganaderas (TECNIGAN), Comité de Ganaderos del Huila, Servicio Nacional de Aprendizaje (SENA). Guía para la implementación de las buenas prácticas ganaderas en sistemas productivos de carne y leche en Colombia. 35 p. 2007.

18. Instituto Colombiano Agropecuario (ICA). Resolución 3585 de 2008. Diario Oficial N. 47.151 de 23 de Octubre de 2008. Bogotá, Colombia. 11 p. 2008. Recuperado 02 Mayo 2016. Disponible En: http://www.icbf.gov.co/cargues/avance/docs/resolucion ica 3585 2008.htm

19. Instituto Colombiano Agropecuario (ICA). Buenas prácticas en el uso de los medicamentos veterinarios y la inocuidad de los alimentos. Ed Produmedios. Bogotá, Colombia. 16 p. 2007.

20. Instituto Colombiano Agropecuario (ICA). Buenas prácticas ganaderas en la producción de ganado bovino y bufalino destinado al sacrificio para el consumo humano. 2016. Recuperado 02 Mayo 2016. Disponible En: http://www.ica.gov.co/getattachment/35f0d70e-b2dd-4bfc-ac1fba169b5ccdca/Publicacion-5.aspx 
21. Instituto Colombiano Agropecuario. Censo pecuario nacional 2016. Recuperado $02 \quad$ Mayo 2016. Disponible En: http://www.ica.gov.co/getdoc/8232c0e5-be97-42bd-b07b9cdbfb07fcac/Censos-2008.aspx

22. Instituto Colombiano Agropecuario. Buenas prácticas ganaderas. 2016. Recuperado $02 \quad$ Mayo 2016. Disponible En: http://www.ica.gov.co/Areas/Pecuaria/Servicios/Inocuidad-en-las-CadenasAgroalimentarias/LISTADO-DE-PREDIOS-CERTIFICADOS-EN-BPG.aspx

23. Instituto Colombiano Agropecuario (ICA). Resolución No. 2341 del 23 de Agosto de 2007. Bogotá, Colombia, 19 p. 2007. Recuperado 02 Mayo 2016. Disponible En: http://www.ica.gov.co/getattachment/0b5de556-cb4a-43a8a27a-cd9a2064b1ab/2341.aspxg

24. Ministerio de Agricultura y Desarrollo Rural (MADR), Ministerio de la Protección Social (MPS), Ministerio de Ambiente, Vivienda y Desarrollo Territorial (MAVDT), Ministerio de Comercio, Industria y Turismo (MCIT), Servicio Nacional de Aprendizaje (SENA), Departamento Nacional de Planeación (DNP). Documento Consejo Nacional de Política Económica y Social (CONPES) 3676 de 2010. Política sanitaria y de inocuidad para las cadenas de la carne bovina y la leche. Bogotá DC, Colombia. 83 p. 2010.

25. Ministerio de Agricultura y Desarrollo Rural (MADR), Ministerio de Protección Social (MPS). Decreto 616, Febrero 28/2006. Bogotá, Colombia. 2006. Recuperado $02 \quad$ Mayo 2016. Disponible En: https://www.invima.gov.co/images/stories/aliementos/decreto 616 2006.pdf

26. Ministerio de la Protección Social (MPS). Decreto 1500 de 2007. Bogotá, Colombia. 41 p. 2007. Recuperado 02 Mayo 2016. Disponible En: http://www.minambiente.gov.co/images/normativa/decretos/2007/dec 150020 $\underline{07 . p d f}$

27. Pezo D, Cruz J, Pinier M. Las escuelas de campo de ganaderos: una estrategia para promover la rehabilitación y diversificación de fincas con pasturas degradadas. Archivos Latinoamericanos de Producción Animal, 15 (1): 42-48. 2007.

28. Uribe F., Zuluaga A.F., Valencia L., Murgueitio E., Ochoa L. Buenas prácticas ganaderas. Manual 3, Proyecto Ganadería Colombiana Sostenible. GEF, BANCO MUNDIAL, FEDEGÁN, CIPAV, FONDO ACCION, TNC. Bogotá, Colombia. 82 p. 2011. 